Let $D_{H_{2}}$ denote a domain containing $H_{2}$ such that $\bar{D}_{H_{2}} \cdot \overline{\left(K+D_{K_{1}}\right)}$ $=0$. Let $D_{K_{2}}$ denote a domain containing $K_{2}$ and such that $\bar{D}_{K_{2}} \cdot \overline{\left(H+D_{H_{1}}+D_{H_{2}}\right)}=0$. This process may be continued and $D_{H}=\sum D_{H_{n}}$ and $D_{K}=\sum D_{K_{n}}$ are two mutually exclusive domains covering $H$ and $K$ respectively.

The University of TeXas

\title{
ON AN INTEGRAL EQUATION WITH AN ALMOST PERIODIC SOLUTION
}

BY B. LEWITAN

We assume that the function $f(x)$ is almost periodic in the sense of $\mathrm{H}$. Bohr and that the functions $E(\alpha), \alpha E(\alpha)$ are absolutely integrable in $[-\infty, \infty]$.

THEOREM. If all real zeros of the function

$$
\gamma(\alpha)=\frac{1}{2 \pi} \int_{-\infty}^{\infty} E(u) e^{-i \alpha u} d u
$$

have integer multiplicities and only two limit points $\infty, \alpha^{*}$, then every solution $\phi(x)$ of the equation

$$
\int_{-\infty}^{\infty} E(\xi-x) \cdot \phi(\xi) d \xi=f(x)
$$

which is uniformly continuous and bounded in $[-\infty, \infty]$ is almost periodic.

Proof. Without loss of generality we may assume that the finite limit point $\alpha^{*}$ has the value 0 ; otherwise we multiply equation (1) by $e^{-i \alpha^{*} x}$.

Putting

$$
f_{n}(x)=\frac{3}{2 \pi} \int_{-\infty}^{\infty} f\left(x+\frac{2 u}{n}\right) \frac{\sin ^{4} u}{u^{4}} d u
$$

we obtain

$$
\int_{-\infty}^{\infty} E(\xi) \phi_{n}(\xi+x) d \xi=f_{n}(x),
$$


where

$$
\phi_{n}(t)=\frac{3}{2 \pi} \int_{-\infty}^{\infty} \phi\left(t+\frac{2 u}{n}\right) \frac{\sin ^{4} u}{u^{4}} d u .
$$

If $v_{n}(\alpha)$ denotes the generalized Fourier transform of $\phi_{n}(t)$, then, in our case, $\dagger v_{n}(\alpha)$ is a linear function for $\alpha>2 n$ and $\alpha<-2 n$.

The functions $f_{n}(x)$ and $\phi_{n}(x)$ are differentiable and the derivative of $\phi_{n}(x)$ is bounded. The function $E(\xi)$ being absolutely integrable, we therefore obtain

$$
\int_{-\infty}^{\infty} E(\xi) \phi_{n}^{\prime}(\xi+x) d \xi=f_{n}^{\prime}(x) .
$$

Putting

$$
\lambda_{\epsilon}(\alpha)=\left\{\begin{array}{cl}
1 & \text { for }|\alpha| \leqq \epsilon, \\
\left(2-\frac{|\alpha|}{\epsilon}\right)^{2}\left(2 \frac{|\alpha|}{\epsilon}-1\right) & \text { for } \epsilon \leqq|\alpha| \leqq 2 \epsilon, \\
0 & \text { for }|\alpha| \geqq 2 \epsilon,
\end{array}\right.
$$

and

$$
\begin{aligned}
\tau_{\epsilon}(u) & =\frac{1}{2 \pi} \int_{-\infty}^{\infty} \lambda_{\epsilon}(\alpha) e^{-i \alpha u} d \alpha, \\
f_{n, \epsilon}(x) & =f_{n}^{\prime}(x)-\int_{-\infty}^{\infty} f_{n}^{\prime}(x+u) \tau_{\epsilon}(u) d u \\
\phi_{n, \epsilon}(x) & =\phi_{n}^{\prime}(x)-\int_{-\infty}^{\infty} \phi_{n}^{\prime}(x+u) \tau_{\epsilon}(u) d u,
\end{aligned}
$$

we obviously have

$$
\int_{-\infty}^{\infty} E(\xi) \cdot \phi_{n, \epsilon}(\xi+x) d \xi=f_{n, \epsilon}(x) .
$$

If $v_{n, \epsilon}(\alpha)$ and $u_{n, \epsilon}(\alpha)$ are generalized Fourier transforms of $\phi_{n, \epsilon}(x)$ and $f_{n, \epsilon}(x)$, then the relation $\ddagger$

† S. Bochner, Mathematische Annalen, vol. 102 (1929), pp. 489-504, vol. 103 (1930), pp. 588-597.

$\ddagger$ S. Bochner, loc. cit. 


$$
\gamma(\alpha) d v_{n, \epsilon}(\alpha)=d u_{n, \epsilon}(\alpha)
$$

holds.

It follows from the construction of the function $\lambda_{\epsilon}(\alpha)$ that the function $\gamma(\alpha)$ has a finite number of zeros in those intervals where $v_{n, \epsilon}(\alpha)$ is not linear. Consequently, by a result of S. Bochner, $\nmid$ the function $\phi_{n, \epsilon}(x)$ is almost periodic in the sense of $\mathrm{H}$. Bohr.

When $\epsilon \rightarrow 0, \phi_{n, \epsilon}(x)$ converges to $\phi_{n}^{\prime}(x)$ uniformly in $[-\infty, \infty]$. This follows from

$$
\int_{-\infty}^{\infty} \phi_{n}^{\prime}(x+u) \tau_{\epsilon}(u) d u=\epsilon \int_{-\infty}^{\infty} \phi_{n}(x+u) \frac{\tau_{\epsilon}^{\prime}(u)}{\epsilon} d u \leqq \epsilon M \rightarrow 0,
$$

where $M$ is a constant. Hence, $\phi_{n}^{\prime}(x)$ is almost periodic in the sense of H. Bohr. But $\phi_{n}(x)$ is bounded. Therefore, by the theorem of Bohr, $\phi_{n}(x)$ is also almost periodic. Finally, $\phi(x)$ being uniformly continuous, the sequence $\phi_{n}(x)$ converges to $\phi(x)$ uniformly in $[-\infty, \infty]$ as $n \rightarrow \infty$, and $\phi(x)$ is almost periodic itself.

We note that the assertion of the theorem remains valid if, more generally, the limit points of the zeros of $\gamma(\alpha)$ are isolated; it is also possible to drop the assumption that the zeros have integer multiplicities.

Mathematical Institute, University A. M. Gorki, CHARKow, U.S.S.R.

† S. Bochner, loc. cit. 